Collection SFN 12 (2011) 247-262

(C) Owned by the authors, published by EDP Sciences, 2011

DOI: $10.1051 / \mathrm{sfn} / 201112012$

\title{
Physics of fluids confined in nanochannels: A shared space for neutrons and simulation
}

\author{
D. Morineau
}

\author{
Institut de Physique de Rennes, CNRS-Université de Rennes 1, France
}

\begin{abstract}
Manipulating fluids at the nanoscale by geometrical confinement is a major issue of contemporary condensed matter science. It is also an area of research where the complementarity of neutron scattering and molecular simulation is particularly apparent. In the present contribution, we consider different cases from the recent literature, where structural (diffraction) and dynamical (quasielastic scattering) investigations by neutron methods can benefit from realistic (atomistic) and coarse-grained molecular simulations, and viceversa. We will emphasize the special benefits and the limitations of each type of approach with the ultimate hope to inspire more scientists to bridge the gap between experiments and simulation.
\end{abstract}

\section{INTRODUCTION}

\subsection{Scientific case}

The significant structural, dynamical and thermodynamical deviations from the bulk properties induced by confinement have been widely addressed by impregnation of molecular fluids within nano/mesoporous structures. For pore sizes smaller than few tens of nanometers, strong interface and finite size effects dominate the static and dynamical properties of the confined phase. Different topics of condensed matter physics have been (re-)considered in confined geometry including H-bonded liquids, polymer physics, phase transitions, critical phenomena, and the glass transition [1]. In the present contribution, we will illustrate the studies of such systems with recent results and achievements made possible by the combination of neutron scattering methods (diffraction and quasi-elastic scattering) and molecular simulation.

\subsection{Mesoporous materials}

The control of the volume of molecular phases at the nanoscale is often obtained by confinement in mesoporous solid materials. Many different types of mesoporous solids have been designed using electrochemical etching, sol-gel chemistry or nanolithography. This allows one to tune parameters such as pore size, pore geometry, surface interaction, and roughness. Figure 1 shows scanning electron microscopy measurements for two prototypical mesoporous materials that have been used in the studies discussed later. The former corresponds to SBA-15 mesostructured porous silicates obtained by hydrothermal condensation of inorganic precursors in the presence of a template agent (surfactant). This method was first described for MCM-41 materials. In both cases, the obtained porosity is formed by cylindrical-like highly monodisperse channels arranged in a triangular crystalline lattice. It is therefore an excellent porous model for experiments in controlled conditions of confinement. The latter is porous silicon (PSi), obtained by electrochemical etching of a silicon wafer in HF solution. It forms a parallel arrangement of not-connected channels of $200 \mu \mathrm{m}$ in length, as shown on the side view. Despite a larger

This is an Open Access article distributed under the terms of the Creative Commons Attribution-Noncommercial License 3.0, which permits unrestricted use, distribution, and reproduction in any noncommercial medium, provided the original work is properly cited. 

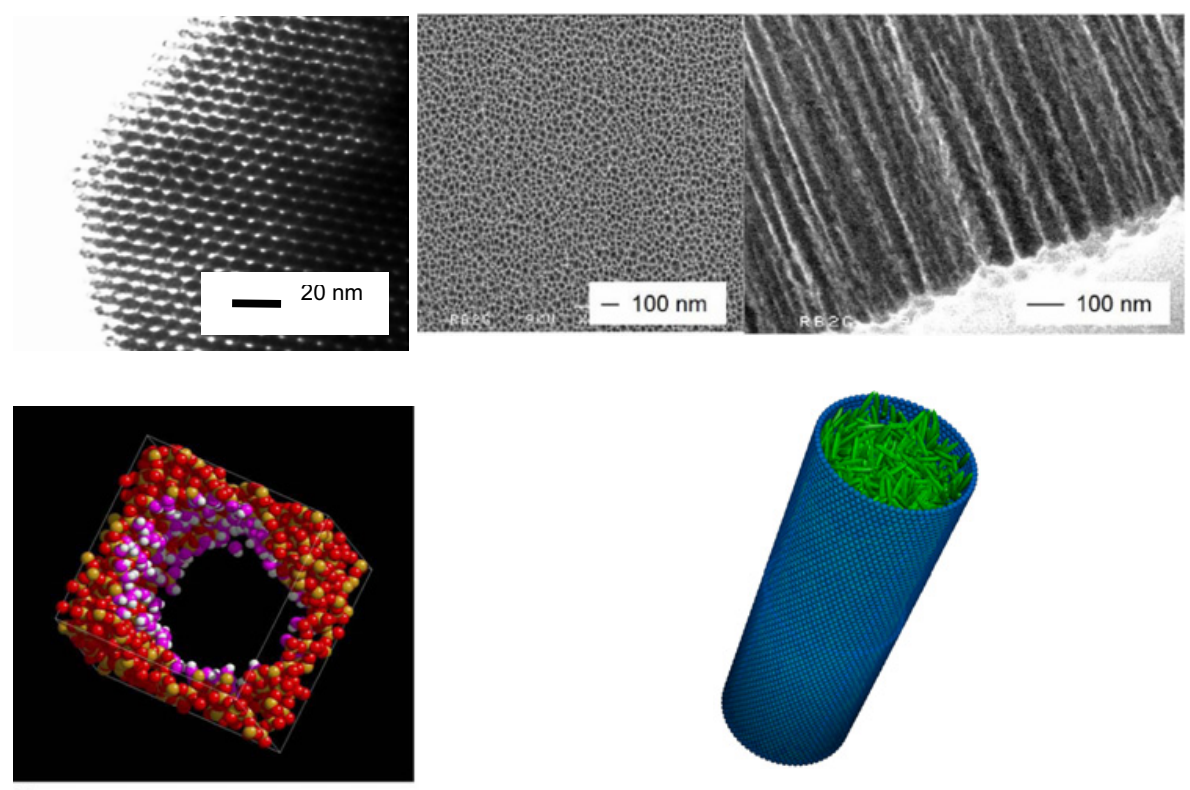

(a)

Figure 1. Electron micrographs and simulation snapshots of different mesoporous materials. Top (real materials): SBA-15 silicate (left) from ref. [2], porous silicon (centre and right) from ref [3]. Bottom (simulated systems): atomistic silica channel (left) from ref. [4], coarse grained channel (right) from ref. [5].

pore-size dispersion, the macroscopic alignment of PSi channels is in some cases advantageous with respect to MCM-41 or SBA-15, which are fine powders.

Different levels of approximation can be used to model the porous channels. Atomistic description provides a more realistic description of the porous structure and the interparticle interaction. It usually implies a large number of interaction sites and it is therefore preferred when a careful account for interfacial interaction is required. This is for instance the case, when interfacial H-bonds are formed (see below the case of alcohols in silicates) and when the hydrophobic character of the surface has to be tuned finely. Conversely, coarse-grained descriptions are much less CPU time demanding and can be used to catch the essential ingredients of some physical phenomena on extended length and time scales (see below the case of phase transitions in liquid-crystals).

\subsection{Methods}

\subsubsection{Structure}

Diffraction is a unique experimental tool to investigate the structure of liquids. Valuable information about the intermolecular short-range order is contained in the static structure factor $S(q)$ or the pair correlation function $g_{L}(r)$ obtained by a Fourier transform analysis, as expressed in Eq. (1):

$$
S(q)=f_{1}(q)+\frac{4 \pi}{q} \rho \int\left(g_{L}(r)-1\right) r \sin (q r) d r
$$

$S(q)$ is usually split into an intramolecular form factor $f_{1}(q)$ and an intermolecular term that contains $g_{L}(r)$ and where $\rho$ is the liquid density [6].

In the case of confined materials, diffraction experiments primarily describe the possible modifications of the phase behavior. Changes of the phase transition temperatures, such as a depression of the freezing point have been often observed. In addition, diffraction experiments tell about the 
character of the phase transitions, which has been shown to be changed from first order to continuous for some confined liquid-crystals [7]. Moreover, diffraction experiments reveal possible changes in the sequence of phases of the confined system with respect to its bulk counterpart. For various systems spanning from water to liquid-crystals, crystalline structures that differ from the normal bulk one can be formed in confinement $[8,9]$. In some cases, these crystalline forms correspond to well-known metastable states of the bulk system, the nucleation of which is favored by confinement. In other cases, these confined crystalline structures have never been observed in the bulk [9]. Moreover, it has been shown for benzene that crystallization can be definitively avoided by confinement. In the latter case, a glass transition from the liquid to an amorphous solid state eventually occurs at much lower temperature $[2,10]$.

The characterization of the structure of the liquid state from neutron diffraction results is more complex in confined geometry than in the bulk state. It has required recent methodological developments, which are described in the following $[11,12]$. Neutron scattering experiment probes the differential cross section, which can be reduced to a composite structure factor $S(Q)$, which includes fluid-fluid and fluid-matrix spatial correlations that are not easily disentangled (cf. Eq. (2)):

$$
\begin{aligned}
S(q)= & f_{1}(q)+X_{\mathrm{Liq}} \frac{4 \pi}{q} \rho \int\left(\left(\tilde{g}^{\mathrm{Liq}}(r)-1\right) g_{u}^{(p p) \text { intra }}(r)+g_{u}^{(p p)}(r)-1\right) r \sin (q r) d r \\
& +2 \frac{\hat{b}_{\mathrm{SiO}_{2}}}{\hat{b}_{\mathrm{Liq}}} X_{\mathrm{SiO}_{2}} \frac{4 \pi}{q} \rho \int\left(\tilde{g}^{\left.\mathrm{Liq}^{\mathrm{L}-\mathrm{SiO}_{2}}(r) g_{u}^{(p w)}(r)-1\right) r \sin (q r) d r}\right.
\end{aligned}
$$

In equation (2), the first term $f_{1}(\mathrm{q})$ is the molecular form factor, while the second and the third terms correspond to the fluid-fluid and fluid-matrix spatial correlations, respectively. $\rho$ is the total number density; $\mathrm{X}_{\mathrm{Liq}}$ and $\mathrm{X}_{\mathrm{SiO} 2}$ are the molar fraction of liquid and silicate units. $\tilde{g}^{\mathrm{Liq}}(r)$ and $\tilde{g}^{\mathrm{Liq}-\mathrm{SiO}_{2}}(r)$ reveal the intrinsic correlations within the fluid and between the fluid and the matrix. The difficulty arises from the fact that these two quantities are modulated by different uniform fluid pair correlation functions, denoted $g_{u}^{(p p) \text { intra }}(r), g_{u}^{(p p)}(r)$ and $g_{u}^{(p w)}(r)$. These there uniform pair correlation functions only depend on the geometry of the porous matrix. More specifically, $g_{u}^{(p p)}(r)$ is defined as the pair correlation function of a system of non-interacting particles (ideal gas) confined in the porous geometry under study. $g_{u}^{(p p) \text { intra }}(r)$ is defined likewise, but the correlations are restricted to non-interacting particles confined in a same pore. Hence, $g_{u}^{(p p) \text { intra }}(r)$ and $g_{u}^{(p p)}(r)$ represent the pore-pore correlations of the porous matrix within the same pore and within the entire porous volume, respectively. This aspect is relevant in the case of porous materials formed by an assembly of disconnected pores, such as MCM-41 molecular sieves. Finally, $g_{u}^{(p w)}(r)$ reflects cross correlations between pairs of non-interacting particles, which can only be present in the porous volume and in the volume defined by the silica walls respectively. Their computation for MCM-41 types of porous geometry is fully detailed in ref. [12]. A central feature of confined systems is the so-called 'excluded volume effect'. It relates the fact that pair correlation functions are sensitive to both the local structure of the phase ('intrinsic intermolecular correlations') and the requirement that a fraction of space is inaccessible to the molecules (out of pore or in pore volume for liquid and silica respectively). For nanoconfined fluids, this effect is always large and should be included in the interpretation of the experiments. If the porous geometry is characterized, it is possible to relate the distortions of the structure factor due to excluded volume effects to the three uniform correlation functions that only depends on the topology of the porous materials as indicated in Eq. (2).

Another usual limitation arises from the fact that neutron scattered intensity is averaged over the entire porous volume. Confinement implies the existence of solid surfaces that enforce some boundary conditions. Consequently, most physical properties of the liquid are expected to lose their translation symmetry. This reduces the understanding of nanoconfined systems, the properties of which are intrinsically heterogeneous inside the pore as shown later in the different examples. 
Molecular simulation provides efficient ways to clarify these issues. Indeed it allows a direct evaluation of the liquid-matrix cross-correlations, which can be fully disentangled from liquid-liquid correlations [13]. Moreover it provides position sensitive properties, which are key elements to characterize interfacial ordering and radial structures properly. Interfacial H-bonds, radial density profiles, local order parameters, and orientation correlations are shown in the following parts to illustrate the inputs from molecular simulation.

\subsubsection{Dynamics}

Quasielastic neutron scattering experiments have been widely used to investigate confinement effects on the molecular dynamics on a typical timescale from 1 picosecond to 10 nanoseconds. The use of fully hydrogenated molecules allows highlighting the incoherent scattering from the confined liquid. Apart from inelastic scattering that occurs at higher energy, the rigid porous matrix essentially contributes as a weakly temperature dependant elastic term. Attention should be paid to mobile surface hydroxyl groups, the contribution of which can be reduced by deuterium exchange.

After subtraction of the matrix contribution, the scattering intensity from the fully hydrogenated molecules essentially corresponds to the incoherent scattering function $S_{\text {inc }}(Q, \omega)$, which allows one to probe the self-correlation of the hydrogen motion [14]. This function is commonly approximated by Eq. (3),

$$
S_{\text {inc }}(q, \omega)=\exp \left(-\frac{\left\langle u^{2}\right\rangle q^{2}}{3}\right)\left[A(q) \delta(\omega)+(1-A(q)) S_{q u a s i}(q, \omega)\right]
$$

In this expression, $A(q)$ stands for the elastic incoherent structure factor coming from restricted motions, $S_{\text {quasi }}(q, \omega)$ is the quasielastic response of slow molecular relaxations, and the contribution of the fast vibrational modes is approximated by the Debye-Waller factor expressed as an overall intensity loss in terms of the vibrational mean square displacement $\left\langle u^{2}\right\rangle$. Note that vibrational modes also contribute at higher energy transfer in terms of inelastic scattering, which is not addressed in the present lecture.

One difficulty in the study of nanoconfined liquids by quasielastic neutron scattering arises from the broadening of the distribution of relaxation times. The decay of relaxation functions usually occurs on 2 or 3 decades in time, which requires the use of many spectrometers to cover a complementary range of timescale. In addition, an upper level of complexity is usually met in nanoconfined liquids because relaxation modes can be spatially inhomogeneous and anisotropic. Therefore, the interpretation of experimental results is often based on phenomenological descriptions or requires the development of ad-hoc models. Such models can be inspired by molecular simulation, which can provide spatially resolved and anisotropic quantities, such as local diffusion coefficients, which will be introduced in the following parts.

\section{EXAMPLES BASED ON STUDIES OF H-BONDED LIQUIDS}

\subsection{Introduction}

H-bonds result from relatively strong and directional interactions between polar groups of a variety of molecules, including alcohols and amines. At variance to their Van der Waals counterparts, H-bonded liquids usually present intermediate range order in the liquid state (i.e. up to a few nanometers). Molecular self-association results from a balance between hydrophobic interactions and H-bonds. Depending on the chemical details of the molecule, three-dimensional networks, chains, rings or clusters have been observed.

Alcohols are expected to be particularly sensitive to confinement. First, intermediate-range-order can be affected by finite size effects, which limit the growth of correlation lengths. Second, strong 

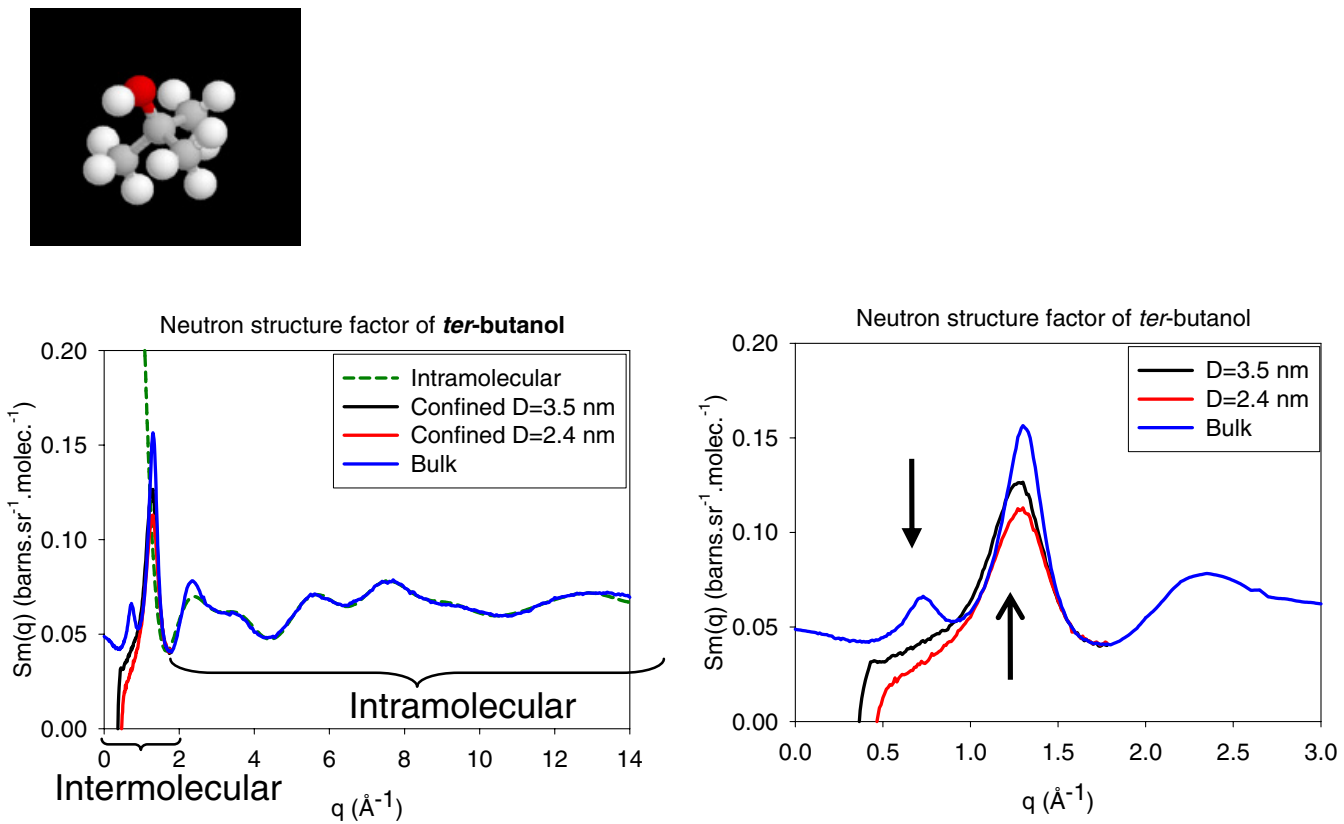

Figure 2. Neutron structure factor of liquid tert-butanol (molecular structure shown in the snapshot) at room temperature in the bulk and confined in MCM-41 silicates with two pore sizes. Left figure: Bulk liquid (blue line), liquid confined in MCM-41 with pore size $D=3.5 \mathrm{~nm}$ (black line), liquid confined in MCM-41 with pore size $D=2.4 \mathrm{~nm}$ (red line), and intramolecular form factor $f_{1}(q)$ (dashed line). Right figure: view of the same quantities in the low- $q$ range in order to magnify the region of the pre-peak $\left(q \approx 0.8 \AA^{-1}\right)$ and the main diffraction peak $\left(q \approx 1.4 \AA^{-1}\right)$. After D. Morineau et al. [18].

liquid-matrix interactions are induced by the formation H-bond bridges between alcohol molecules and surface silanol groups of hydrophilic porous silica materials.

\subsection{Structure of nanoconfined H-bonded liquids}

A paradigm of mesoscopic H-bond-induced clustering is provided by globular alcohols. These systems are known to spontaneously form some long-lived micelle-like clusters of a few molecules in the liquid state. The cluster formation results from the competition between strong and directional H-bond interactions of polar hydroxyl groups and the repulsion between the hydrophobic bulky parts of the molecules. On the basis of molecular simulations and X-ray and neutron scattering experiments, it has been shown that the formation of these microscopic arrangements has a clear experimental signature in terms of a pre-peak in the static structure factor [15]. Tert-butanol $\left(\left(\mathrm{CH}_{3}\right)_{3}-\mathrm{COH}\right)$ is probably the simplest molecule among the variety of other systems, that display H-bonded clustering, such as aromatic amines like $\mathrm{m}$-toluidine and $\mathrm{m}$-fluoroaniline, or aromatic alcohols like m-cresol [16, 17]. For this reason and thanks to its globular form, it is so far also one of the most studied associating liquids and can be considered as a model system for studying the generic features of mesoscopic H-bond-induced clustering.

Figure 2 shows the structure factor of liquid tert-butanol. It is dominated by intramolecular contributions for $q$-values above $4 \AA^{-1}$ (i.e. the form factor $f_{1}(q)$ in eq. (1)). Intermolecular contributions appear as a main diffraction peak $\left(q=1-1.8 \AA^{-1}\right)$ that mostly reflects short-range-order and a pre-peak centered at about $q=0.8 \AA^{-1}$ that reflects supermolecular assemblies. In confinement, the main diffraction peak is broadened and its total intensity is strongly reduced (by about $30 \%$ ). 

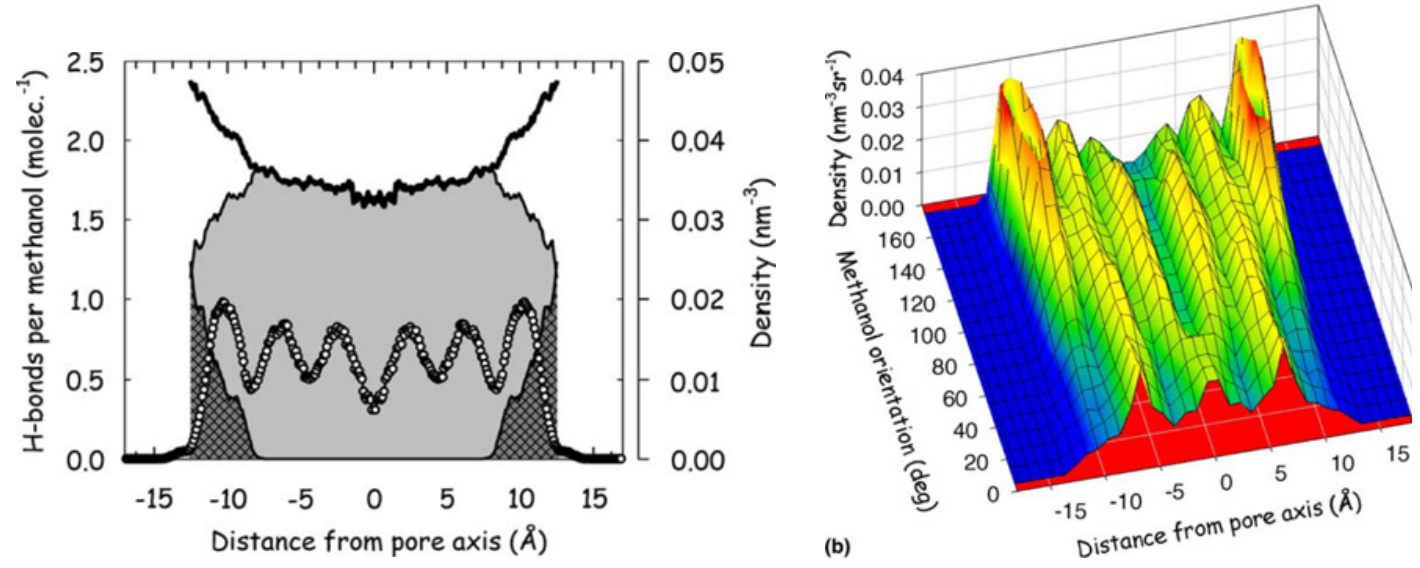

Figure 3. Radial density profile of methanol confined in a silica channel. (a) Open circles: local number density of methanol molecules. Dark grey shaded area: average number of heteromolecular H-bonds, bounding one methanol and one surface silanol. Light grey shaded area: average number of homomolecular H-bonds, bounding two methanol molecules. Solid line: average number of H-bonds per methanol molecule, including both heteromolecular and homomolecular types of H-bonds. (b) Radial density profile as a function of molecular orientation with respect to the pore surface. Monte Carlo simulation results after R. Guégan et al. [4].

The second major effect concerns the pre-peak, which disappears under confinement. Note that other differences at lower $q$ values, i.e., a negative intensity for $q<0.5 \AA^{-1}$, are related to the mesoporous structure. The large changes of $S(q)$ in the region of the pre-peak and the main diffraction peak suggest that the local order of the liquid phase is fundamentally disrupted by nanoscale confinement. More specifically, the absence of any correlation peak at the position of the pre-peak means that supermolecular clusters, which are an essential ingredient of tert-butanol are unstable in nanopores.

Different reasons can be invoked to interpret the absence of pre-peak in the structure factor of nanoconfined tert-butanol. Supermolecular self-assembly in H-bonded liquids results from a delicate balance between hydrophobic and H-bond interactions, which has been shown to be sensitive to external parameters such as temperature or pressure. The spatial restriction introduced by the porous geometry imposes severe constraints on the extension of medium-range ordered structures. Such effect does not only depend on the pore size (finite size effect) but also on the details of the pore shape. Moreover, the proximity of a solid surface often promotes specific organizations in the interfacial fluid, such as layering, linear aggregates, or hexatic order (i.e. sixfold orientational order). The ordering of the interfacial fluid can compete with the inherent structures of the bulk liquid. In principle, such interactions between the fluid and the matrix should show up in the neutron structure factor but are not easily accessible.

Molecular simulation should be performed to have a better insight on interfacial order. Layering is a well-known feature that illustrates spatial inhomogeneity of the density in fluids confined between two surfaces approached at the nanometer scale. As an example, Fig. 3(a) shows the radial density of the carbon atoms of methanol across the pore diameter. It clearly shows oscillations around the average usual density of the liquid $\left(\rho=0.014 \mathrm{~nm}^{-3}\right)$. The three concentric shells have a regular spacing, which corresponds to one molecular diameter. The amplitude of the density fluctuations $\pm 35 \%$ is not significantly damped in the centre of the pore for this pore diameter. The formation of layers in terms of a non-homogeneous distribution of the liquid density within the pore reflects the propagation of translational order from the wall surface towards the pore center. It breaks the translational invariance of the system, which is, on the contrary, an intrinsic feature of normal bulk liquids. Another specific feature of confined methanol concerns the orientational order, as shown in Fig. 3(b). Normal liquids are 


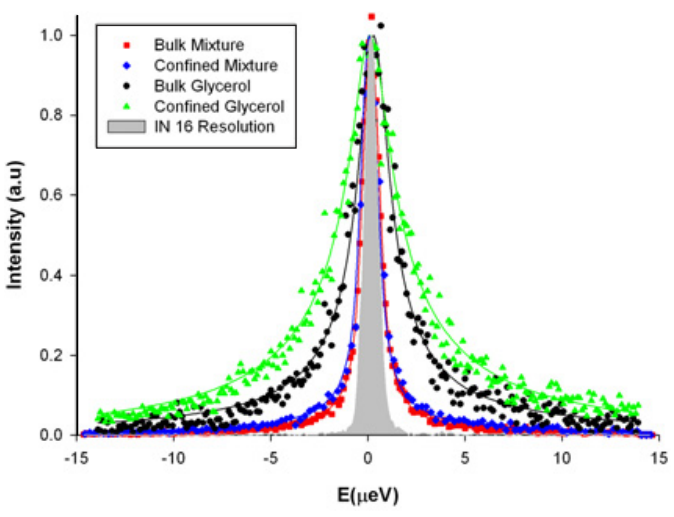

Figure 4. Dynamic structure factor by incoherent quasielastic neutron scattering of glycerol and a trehaloseglycerol bioprotectant solution in the bulk and confined in nanoporous silicon at $T=310 \mathrm{~K}$. The spectra have been recorded at the same value of the transfer of momentum $q=0.96 \AA^{-1}$. The curves are normalized to maximum intensity. After R. Busselez et al. [3].

isotropic, which implies that on average each molecular dipole can point towards any particular direction of space with the same probability. At variance, confined methanol loses such a spherical symmetry. This orientational order is characterized in Fig. 3(b), which shows the radial density profile as a function of the orientation of the molecular dipole with respect to the pore surface. Obviously, the contact layer is anisotropic, with a maximum corresponding to the molecular axis being normal to the surface.

\subsection{Dynamics of nanoconfined H-bonded liquids}

The dynamics of H-bonded liquids is illustrated by the study of glycerol and trehalose-glycerol glassforming solutions [3]. The incoherent scattering function has been measured for the two liquids in the bulk and confined in nanoporous silicon as shown in Fig. 4. The incoherent scattering function appears essentially as a quasielastic line, which is broader than the elastic resolution $\left(A(q)=0, S_{\text {inc }}(q\right.$, $\omega) \propto S_{\text {quasi }}(q, \omega)$ in eq. (3)). The absence of any purely elastic contribution proves the loss of correlation of the protons motion on the time scale of the experiment. This non-localized character of the motion of the molecules most probably implies that translational diffusion processes occur on the time and length scales of the experiment. Comparing the line width of the scattering functions, one can also deduce the qualitative conclusion that the relaxation dynamics of the pure glycerol samples is faster at $310 \mathrm{~K}$ than for the binary solution samples. Nevertheless, a more quantitative analysis of the shape of the quasielastic lines is required to draw more detailed conclusions.

The scattering functions have been fitted at each value of the transfer of momentum $q$ by the Fourier transform of a Kohlrausch function, weighted by a Debye-Waller factor $f(q)$ and convoluted with the experimental resolution $R(\omega)$ according to equation (4):

$$
S_{\text {quasi }}(q, \omega)=f(q) R(\omega) \otimes F T\left[\exp \left(-\left(\frac{t}{\tau_{K}(q)}\right)^{\beta_{K}}\right)\right]
$$

This stretched-exponential law is recognized as a convenient empirical way to reproduce the relaxation functions of glass-forming materials. This single function usually better reproduces the experimental data than a discrete combination of different modes (translation, rotation ...), which are most probably coupled in the case of glass-forming liquids $[19,20] . \tau_{K}$ stands for the characteristic relaxation time, whereas the exponent $\beta_{K}$ reflects the non-Debye character of the relaxation function. Small values of $\beta_{K}$ 

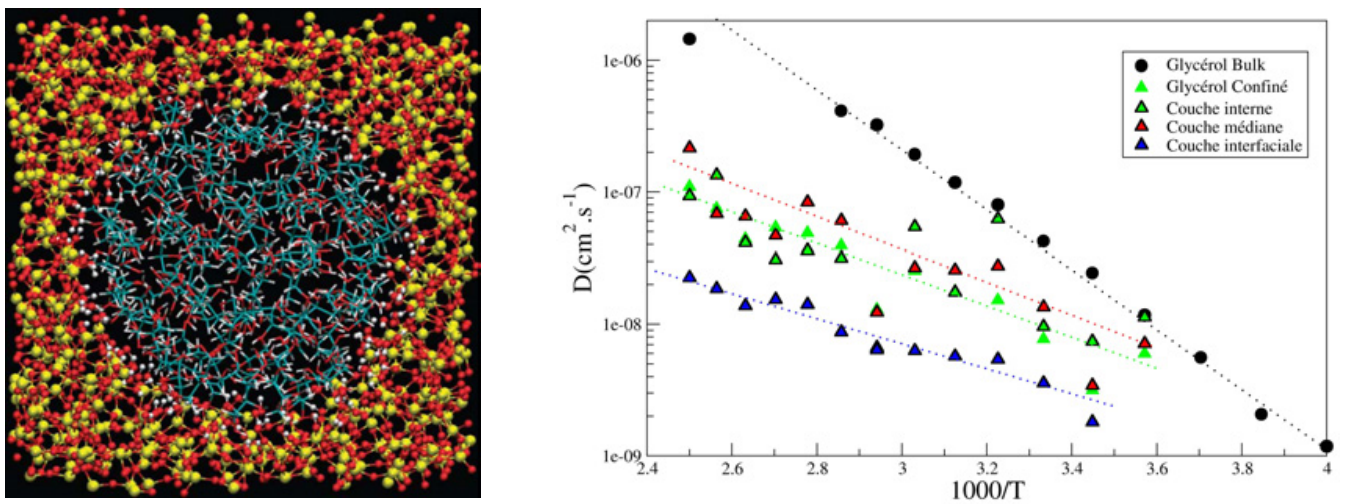

Figure 5. (Left) Snapshot of liquid glycerol confined in a silica channel. (Right) Local values of the diffusion coefficients of confined glycerol for the three different layers compared with the bulk one. After R. Busselez et al. [25].

express a significant departure from a simple exponential decay, whereas a Debye process is recovered for $\beta_{K}=1$.

The value of the $\beta_{K}$ exponent for pure glycerol is about 0.6 . Interestingly, $\beta_{K}$ differs significantly for the binary solution $\left(\beta_{K}=0.5\right.$ for the bulk binary solution) and is also reduced by confinement, being equal to $\beta_{K}=0.5$ and $\beta_{K}=0.4$ for the pure confined glycerol and the confined binary solution, respectively.

It is well-known that the stretched exponential relaxation function may be expressed within two different scenarios, corresponding to the limiting cases of heterogeneous and homogeneous dynamics [21]. The homogeneous scenario assumes that the relaxation process is intrinsically of non-Debye type. On the contrary, the heterogeneous scenario relates stretching to the existence of dynamical heterogeneities of given typical size and life-time. Each individual heterogeneity may relax according to a simple Debye mode, with a different relaxation time, leading on average to a broadening of the distribution of relaxation times.

Confinement leads to a significant reduction of the $\beta_{K}$ exponent. This increase of the non-Debye character of the relaxation is likely to be attributed to the formation of spatial heterogeneities within the pore, the dynamical parameters of which depend on the distance to the pore surface. There is indeed an increasing number of experimental and numerical evidence that the dynamics of a confined fluid is spatially heterogeneous [22-24]. The case of the confined trehalose-glycerol solution is very striking, leading to a very small $\beta_{K}$ exponent $\left(\beta_{K}=0.4\right)$. For a binary glass-forming liquid, it is not excluded that dynamical heterogeneities can be amplified by a non-homogeneous concentration distribution profile across the pore diameter.

Indeed, molecular dynamics simulations of glycerol provide a direct insight into dynamical heterogeneities [25]. The computed values of the diffusion coefficients as a function of the inverse temperature (Arrhenius representation) are shown in Fig. 5. The diffusion coefficients have been measured locally and averaged over the three different layers defined by the radial density profile.

\section{EXAMPLES BASED ON STUDIES OF LIQUID-CRYSTALS}

\subsection{Introduction}

Liquid crystals (LCs) in confined geometry are extensively utilized for various technological applications, including displays, optical switches and many other electro-optical devices. Moreover, the physics of confined LCs reveals several original features, many of which are still open questions 


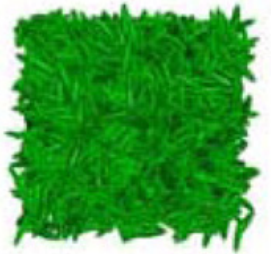

Isotropic

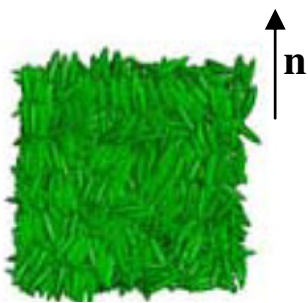

Nematic

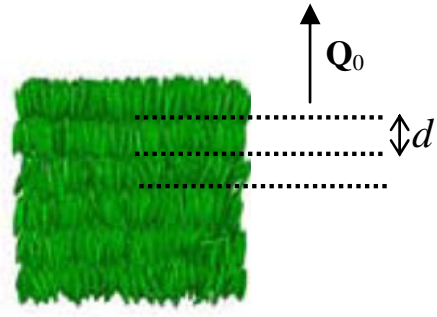

Smectic

Figure 6. Snapshots illustrating the typical structures observed in liquid-crystals in the isotropic, nematic, and smectic phases (from left to right). In the nematic phase, the presence of orientational order is characterized by a preferential direction of alignment of the molecular axis along the nematic director $\mathbf{n}$. In the smectic phase, the formation of layers is characterized by the interlaying spacing $d$, which is the typical period of density modulation along the wave vector $\mathrm{Q}_{0}$. After Q. Ji et al. [26].

fundamentally, such as the theoretical treatment of phase behaviors under external fields induced by confinement or the interfacial properties of soft-matter and biomimetic materials. LCs present anisotropic intermediate phases, also called mesophases, with partial orientational and positional orders. Orientational order means that the molecular axes point in a particular direction, whereas positional order means that the molecular centers occupy sites on a 1,2 or 3D periodic lattice. An important class of LCs is formed by rod-like (calamitic) molecules.

One of the most rigorously studied calamitic LCs in confined geometry is 4-n-octyl-4'cyanobiphenyl $(8 \mathrm{CB})$. In the bulk, its sequence of phases obtained with increasing temperature is crystal $(\mathrm{K})$, smectic $\mathrm{A}(\mathrm{A})$, nematic $(\mathrm{N})$ and isotropic $(\mathrm{I})$ with the following transition temperatures: $\mathrm{T}_{\mathrm{KA}}=294.5 \mathrm{~K}, \mathrm{~T}_{\mathrm{NA}}=306.3 \mathrm{~K}$ and $\mathrm{T}_{\mathrm{NI}}=313.5 \mathrm{~K}$.

The nematic phase is characterized by a preferential orientation of the main molecular axis along the nematic director, as sketched in Fig. 6. The orientational order parameter $S$ is defined according to the following equation, where $\mathbf{n}$ is the nematic director, $\hat{\mathbf{u}}_{i}$ is the unit vector along the principal axis of the molecule $i$ and \langle\rangle$_{i}$ stands for the average over the particles $i$ :

$$
S=\left\langle 3\left(\hat{\mathbf{u}}_{i} \cdot \mathbf{n}\right)^{2}-1\right\rangle_{i} / 2
$$

The smectic phase is characterized by the occurrence of periodic modulations of the density in one direction. The molecules form layers, with an additional orientational order within the layer and no long range positional order in the 2 directions perpendicular to the smectic director. The translational order parameter $\tau$ measured along the director for the wave vector $Q_{0}=2 \pi / d$, is defined by:

$$
\tau=\left|\left\langle\exp \left(i \frac{2 \pi}{d} z_{i}\right)\right\rangle_{i}\right|
$$

where $d$ is the interlayer spacing and $z_{i}$ is the ordinate of the centre of the particle $i$. The value of the translational order parameter $\tau$ can be obtained from X-ray and neutron diffraction experiments.

LCs are soft materials with long-range order, and as such they are considerably influenced by geometric confinement, the nature of the porous surfaces, the pore dimensionality and quenched disorder effects imposed by the pore wall morphology [27]. Under confinement, the surface interaction acts as an external field that couples to the LCs order parameters. This effect may induce partial order at the pore wall, which persists well above the clearing temperature [7, 28]. Moreover, the phase behavior is considerably affected by spatial confinement, as well as interfacial effects [28-30]. 


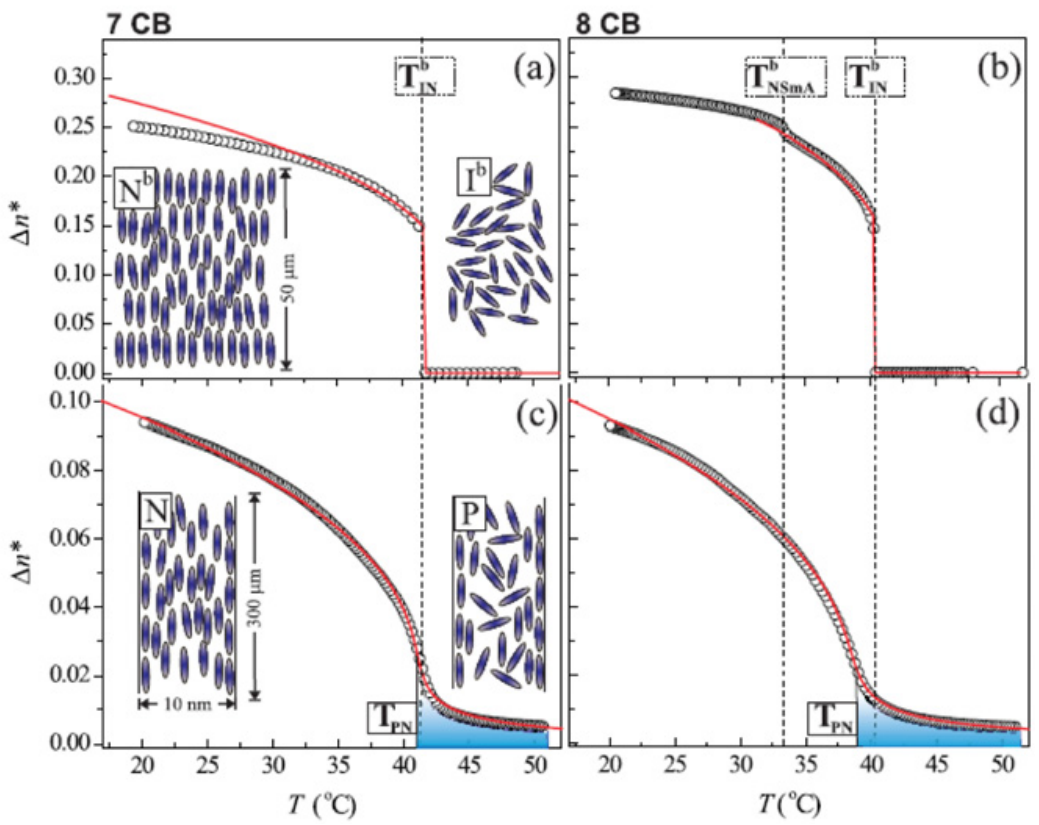

Figure 7. Optical birefringence of 7CB (4-n-heptyl-4'-cyanobiphenyl) and 8CB (4-n-octyl-4'-cyanobiphenyl) measured in the bulk state, panels (a), (b), and in the silica nanochannels, panels (c) and (d), resp., as a function of temperature in comparison to fits (solid lines) based on the KKLZ model. The final birefringence characteristics of the paranematic phases are shaded down to the P-N "transition" temperatures, $\mathrm{T}_{P N}$. The dashed lines mark the bulk I-N and N-SmA transition temperatures. After A. Kityk et al. [28].

\subsection{Structure of nanoconfined liquid-crystals}

The use of transparent monolithic free-standing membranes obtained from fully oxidized porous silicon has allowed unprecedented direct measurements of the nematic order parameter from optical birefringence measurements [7]. This is illustrated in Fig. 7, which shows optical birefringence measurements by Kityk et al. [28]. In this case, LCs were confined within unidirectional straight nanochannels of $10 \mathrm{~nm}$ in diameter and $300 \mu \mathrm{m}$ in length. Under such circumstances, the unidirectional interaction between the pore and the confined LC tends to align the director along the main axis. The LC develops a paranematic order above $T_{I N}$. On cooling, the bulk $I-N$ first order transition is changed to a continuous Paranematic to Nematic transition $(P N-N)$ in confinement.

The effect of surface interaction on the nature of the transition in nanoconfined geometry has been discussed by Kutnjak et al. within the framework of the KKLZ model (Kutnjak-Kralj-Lahajnar-Zumer) derived from the Landau-de Gennes theory [31]. It predicts that in confinement the $I-N$ transition is weakened and its character changes from a strong-first order transition in bulk to a weak first-order or continuous paranematic-to-nematic $(\mathrm{P}-\mathrm{N})$ transition, depending on the strength of surface interaction. The predictions for the temperature variation of the orientational order parameter shown as solid lines in Fig. 7 are in quantitative agreement with experiments.

Smectic order of nanoconfined $8 \mathrm{CB}$ has been analyzed in detail by neutron diffraction and small angle neutron scattering $[9,29]$. Rocking curve measurements have shown that the smectic layers are aligned perpendicular to the pore axis. This results from the strongly unidirectional character of the confining geometry and it is in agreement with the previously mentioned axial planar configuration of the director in the nematic phase.

More interestingly, the width of the smectic Bragg peak is not resolution limited and its intensity grows only gradually on cooling. The gradual growth of the intensity of the smectic Bragg peak at 


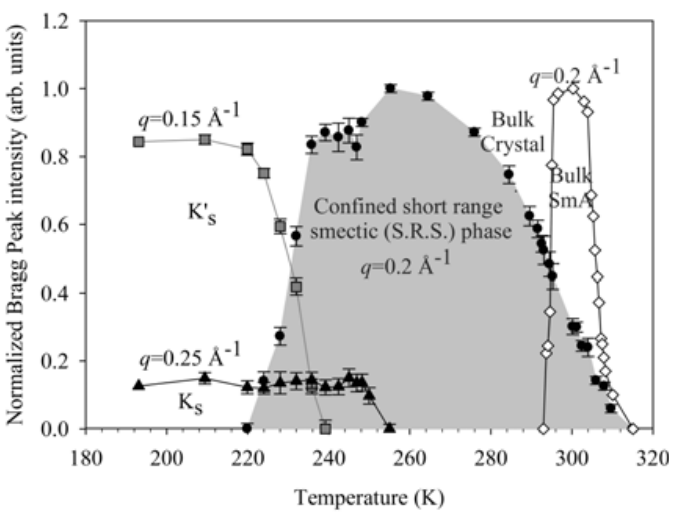

Figure 8. Phase sequence of $8 \mathrm{CB}$ confined in porous silicon $D=8 \mathrm{~nm}$ (filled symbols) during cooling obtained from the temperature variation of the neutron diffraction maximum intensity of a selection of characteristic Bragg peaks of the smectic $\left(q=0.2 \AA^{-1}\right)$, and crystalline $\mathrm{K}_{s}^{\prime}\left(q=0.15 \AA^{-1}\right)$ and $\mathrm{K}_{s}\left(q=0.25 \AA^{-1}\right)$ phases. The smectic region of bulk $8 \mathrm{CB}$ is added for comparison (open diamonds). After R. Guégan et al. [29].

$q=0.2 \AA^{-1}$ as a function of temperature is shown in Fig. 8 (filled circles). The formation of short range smectic order occurs over an extended temperature range from $325 \mathrm{~K}$ down to $260 \mathrm{~K}$. The domain of existence of this short range smectic phase (see shaded area) is limited at low temperature by the nucleation of two crystalline phases (denoted $\mathrm{K}_{s}$ and $\mathrm{K}_{s}$ ), which are not usually obtained in the bulk. This overall behavior contrasts with the sharp increase of long range smectic order observed in the bulk (open circles). The peculiar nature of the smectic ordering of $8 \mathrm{CB}$ in confinement has been rationalized within the framework of random field theories by taking into account quenched disorder effects induced by the internal rough surface of PSi [29].

Molecular dynamics simulations have been performed using a coarse-grained description of both the molecules and the pore [5, 26, 32]. Indeed in the present case, the atomic details of the molecules are not necessary to catch the essential characteristics of the observed phenomena. The interactions between LC molecules are expressed by the pair potential proposed by Gay and Berne for axially symmetric particles. The pore walls were created by decorating the surface of the pores with Lennard-Jones (LJ) atoms in close contact and arranged on a triangular array (cf. Fig. 9).

The temperature variation of the orientational order parameter $S$ of the three confined systems and the bulk is shown in Fig. 9. The bulk isotropic to nematic (I-N) and nematic to smectic A (N-SmA) transition temperatures are specified. It is obvious that $S$ is non-zero even well above the clearing temperature. In the bulk it is about $S=0.05$ at $T=1.9$, which is commonly attributed to finite size effects in the literature. More interestingly, much higher values are observed for the slab and cylinder geometries, respectively, 0.2 and 0.35 at $T=1.9$. It is ascribed to the effect of the surface interaction, which couples to the nematic order parameter, in agreement with experiments. On decreasing the temperature, the orientational order parameter progressively increases for the slab and cylinder geometries, which demonstrates the formation of an ordered nematic domain. The rather continuous variation of $S$ for these two porous geometries contrasts with the quite sharp increase initiated at $T=1.4$ for the bulk. This feature confirms that slab and cylindrical confinement transforms the abrupt I-N bulk transition to a gradual paranematic to nematic $(\mathrm{PN}-\mathrm{N})$ ordering. The order parameter in spherical confinement shows a qualitatively different trend. It remains small in the whole temperature range and exhibit only a modest temperature variation. This suggests that the spherical symmetry of the order formed at the pore surface inhibits the formation of a homogeneous nematic domain. Similar conclusions can be made for the translational order parameter, in qualitative agreement with neutron diffraction experiments. 

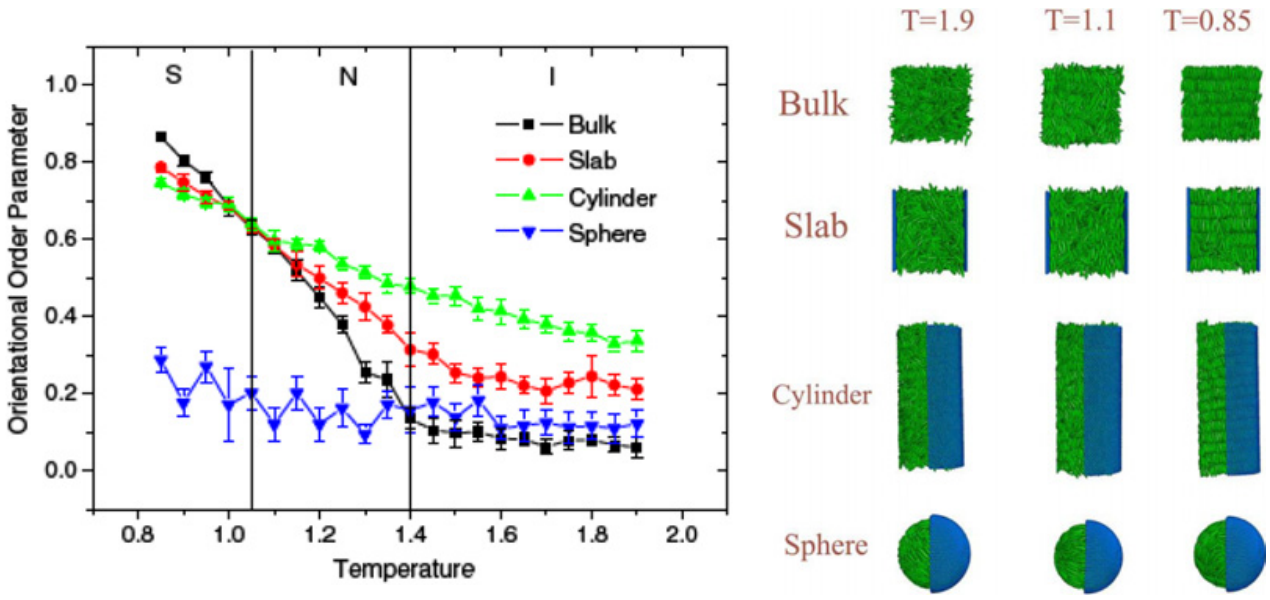

Figure 9. (Left) Temperature variation of the order parameters of a simulated Gay-Berne liquid crystal in the bulk and confined in different porous geometries. The temperature is given in standard reduced unit of $\varepsilon_{\mathrm{w}} / \mathrm{k}_{B}$, where $\mathrm{k}_{B}$ is the Boltzmann constant and $\varepsilon_{0}$ is the energetic parameter of the interaction potential of the Gay-Berne model. (Right) Snapshots of the bulk and confined LC for temperatures corresponding to the different characteristic phases. After Q. Ji et al. [28].

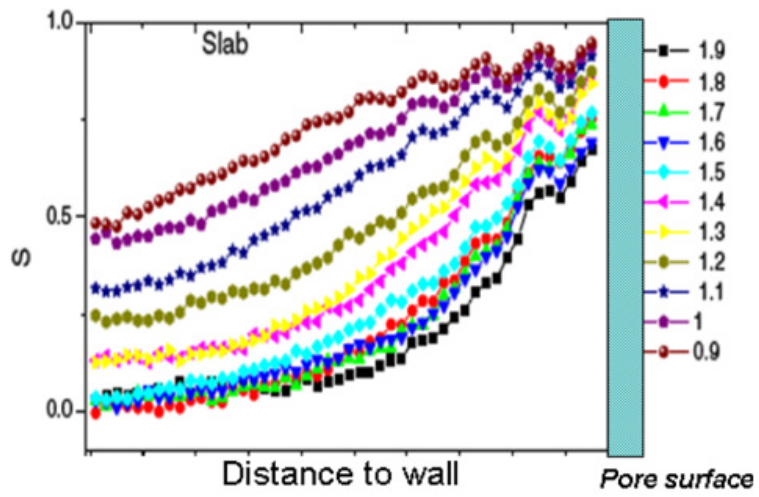

Figure 10. Radial profile of the orientational order parameter of a simulated Gay-Berne liquid crystal in confined geometry as a function of temperature. The temperature is given in standard reduced unit like in Fig. 9. After Q. Ji et al. [26].

Figure 10 shows the radial profile of the orientational order parameter, which has been defined locally as a function of the distance to the pore surface. The influence of the surface induced paranematic order propagates gradually towards the pore centre. At high temperature $T=1.9, S$ reaches a null limit for the three different systems. It means that, despite a strong surface planar anchoring, the centre region of the confined phase remains isotropic. On decreasing temperature, a uniform nematic order progressively develops from the pore surface in the slab and cylinder pores.

\subsection{Dynamics of nanoconfined Liquid-Crystals}

Concerning dynamics, some striking similarities between confined LCs and glass-forming systems are reported in many studies, such as a non-Debye character of the relaxation decay and a non-Arrhenius temperature dependence of the relaxation time [33-35]. 


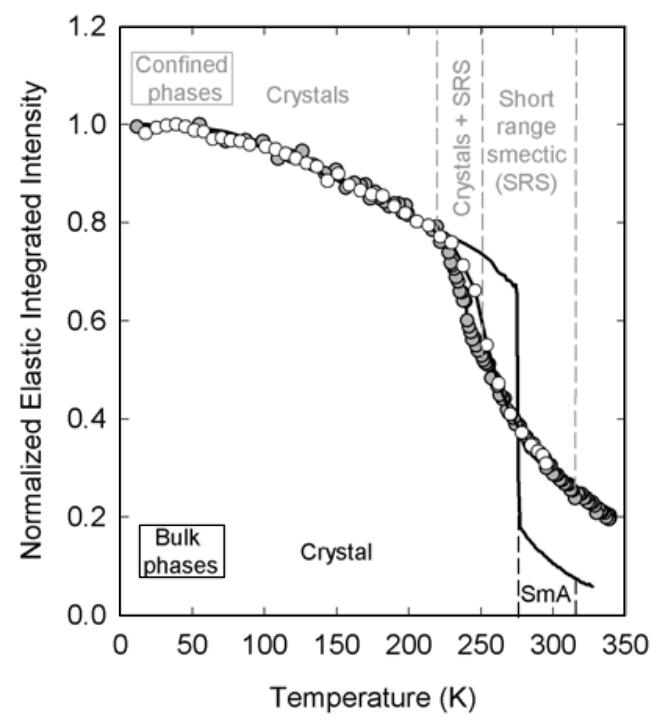

Figure 11. Elastic fixed window scans measured by incoherent neutron backscattering on bulk $8 \mathrm{CB}$ (solid line) and 8CB confined in PSi on cooling (filled symbols) and heating (open symbols). After R. Guégan et al. [34].

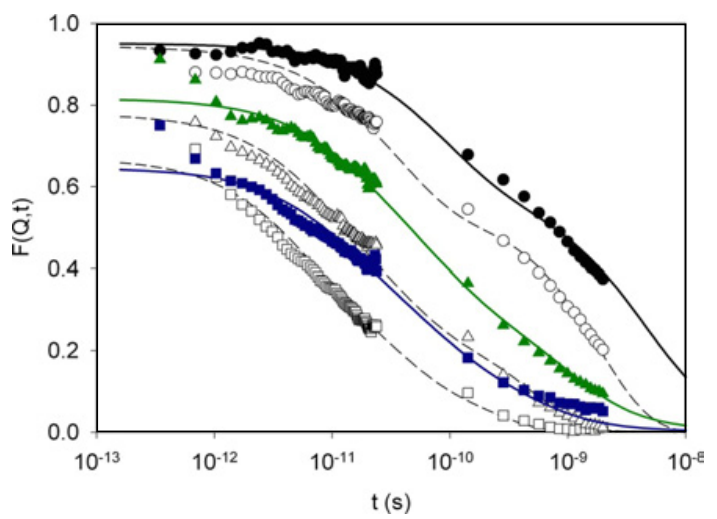

Figure 12. Intermediate incoherent neutron scattering functions of bulk $8 \mathrm{CB}$ (open symbols) and $8 \mathrm{CB}$ confined in PSi at $296 \mathrm{~K}$ (solid symbols) for different values of the transfer of momentum (Q). Lines result from fitting with a heterogeneous dynamics model (see text). After R. Lefort et al. [33].

Elastic fixed window neutron backscattering experiments on $8 \mathrm{CB}$ confined in silicon nanochannels are shown in Fig. 11. In an elastic fixed window scan (EFWS) the measured intensity is, in principle, $S(q, \omega=0)$. It actually integrates all purely elastic or quasielastic contributions narrower (i.e. slower) than the energy resolution defining the "fixed window" $(0.9 \mu \mathrm{eV}$ FWHM). The observation of a remaining elastic component at high temperature well above the clearing point (transition to the isotropic phase) reveals that the dynamics is already significantly reduced by confinement in the liquid phase. This is at variance with the bulk dynamics, which is dominated by a fast translational diffusion mode. On cooling, a continuous glass-like slowdown of the relaxation modes is observed. Again, this differs from the bulk behavior, which exhibits a clear dynamic transition from fluid to solid phases.

The intermediate scattering functions of confined $8 \mathrm{CB}$ display a very pronounced stretching character towards longer times (cf. Fig. 12), which is the signature of the highly non-Debye nature of the 


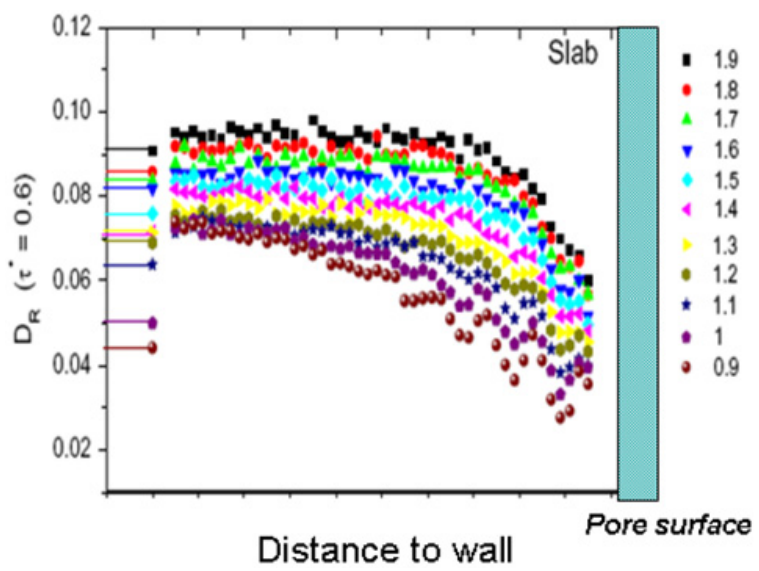

Figure 13. Radial profile of the local rotational diffusion coefficient of confined Gay-Berne liquid-crystal. After Q. Ji et al. [32].

relaxation processes. Both experiments and simulation have associated this feature with an interfacial slowing down effect due to the hindered rotation and diffusion of the surface anchored molecules. This surface effect propagates into the pore volume, leading to a spatially strongly heterogeneous dynamics.

A model taking this heterogeneity into account and based on simple hypothesis has been used by Lefort et al. [33]. It assumes that i) the surface dynamics is frozen within the experimental resolution $(r=R)$, ii) far from the surface, the dynamics tends to that of the bulk, following a simple exponential decay $\frac{1}{\tau(r)} \propto \exp \left(\frac{R-r}{\xi_{\text {wall }}}\right)$ where $\frac{1}{\tau(r)}$ is the inverse local relaxation time at a distance $r$ from the pore center, $R$ is the pore radius, and $\xi_{\text {wall }}$ is the dynamical correlation length that characterizes the range of influence of the surface on the molecular dynamics and iii) the relaxation modes remain qualitatively the same as in the bulk. The input dynamic parameters for the bulk (diffusion coefficient $D$, rotational correlation time $\tau$ and Debye-Waller factors) were obtained from a previous study of unconfined 8CB, where the intermediate scattering functions were fully analyzed as a combination of vibrational modes, uniaxial rotation and centre-of-mass translational diffusion [36]. A local intermediate scattering function $f(r, Q, t)$ is then defined as a function of the distance $r$ to the pore wall according to eq. (7). The total intermediate scattering function $F_{\text {het }}\left(Q, t, \xi_{\text {wall }}\right)$ is obtained by volume average over the entire pore radius $R$ according to Eq. (8). This approach gives reasonable fits of the experimental data (see lines in Fig. 12) and allows one to obtain the value of the dynamical correlation length $\xi_{\text {wall }}$ that measures the spatial extension of the surface effect on the tagged-particle dynamics:

$$
\begin{gathered}
f(r, Q, t)=e^{-Q^{2}\left\langle u_{\text {conf }}^{2}\right\rangle} F^{r o t}\left(\tau_{c}(r), Q, t\right) e^{-D(r) Q^{2} t} \\
F_{\text {het }}\left(Q, t, \xi_{\text {wall }}\right)=\frac{2}{R} \int_{0}^{R} f(r, Q, t) r^{2} d r
\end{gathered}
$$

This heterogeneous model of the dynamics is strongly supported by molecular dynamics computations within the Gay-Berne potential. The radial profile of the rotation diffusion coefficient $D_{R}$ is shown in Fig. 13. It provides a better insight into the local dynamics of the confined phase, which is strongly inhomogeneous. The molecular rotation close to the wall is about five times slower than at the pore centre. The influence of the surface gradually vanishes and a bulk-like plateau value is reached at the pore centre in the almost isotropic phase. On decreasing temperature, the dynamics of the core part of the confined phases, deviates from the bulk one. It results from the extreme broadening of the 
(I-N) transition in confinement. More important, the influence of the surface is mediated towards larger distances on decreasing temperature and eventually affects the entire confined phase.

\section{CONCLUSION}

In the present chapter, we have addressed the very peculiar behaviors of nanoconfined molecular phases. It is striking that many fundamental properties of confined systems, in terms of phase behavior, structure or molecular dynamics significantly depart from their bulk counterparts. It implies that some areas of condensed matter physics have to be re-considered at the nanoscale.

This is obviously one scientific case where the combination of neutron scattering and molecular simulation can be especially fruitful. We have provided a series of examples, where structural (diffraction) and dynamical (quasielastic scattering) investigations by neutron methods can benefit from realistic (atomistic) and coarse-grained molecular simulations.

The ultimate idea that we have tried to emphasize in this contribution is the intrinsic difficulty to account for the extremely heterogeneous nature of nanoconfined systems. It requires a very careful handling of the experimental data, which are spatial averages over heterogeneities. It includes the account for excluded volume effects, the combination of quasielastic spectrometers to attain broadband dynamical range and the development of heterogeneous models of the intermediate scattering function. In this scheme, molecular simulation can provide a unique view of the nanoconfined phase from the computation of spatially resolved (local) structural and dynamical properties.

\section{Acknowledgements}

This manuscript has benefited from fruitful discussions and collaborations with the groups of C. Alba-Simionesco, J.-M. Zanotti, B. Frick, and P. Huber. Examples have been obtained from published results with principal contributions from R. Lefort, A. Ghoufi, Q. Ji, R. Guégan, R. Busselez, and G. Chahine.

\section{References}

[1] See recent reviews : C. Alba-Simionesco et al., J. Phys.: Condens. Matter 18, R15 (2006); M. Alcoutlabi and G. B. McKenna, J. Phys.: Cond. Mat. 17, R461 (2005).

[2] G. Dosseh, N. Brodie-Linder, B. Frick, C. Le Quellec, D. Morineau and C. Alba-Simionesco, Ann. Chim. Sci. Mat. 30, 365-373 (2004).

[3] R. Busselez, R. Lefort, M. Guendouz, B. Frick, O. Merdrignac-Conanec, and D. Morineau, J. Chem. Phys. 130, 214502 (2009).

[4] R. Guégan, D. Morineau and C. Alba-Simionesco, Chem. Phys., 317, 236-244 (2005).

[5] Q. Ji, R. Lefort, R. Busselez, and D. Morineau, J. Chem. Phys., 130, 234501 (2009).

[6] J. G. Powles, Adv. in Physics 22, 1 (1973).

[7] G. Chahine, A.V. Kityk, K. Knorr, R. Lefort, M. Guendouz, D. Morineau, and P. Huber, Phys. Rev. E, 81, 031703 (2010).

[8] M.-C. Bellissent-Funel, J. Lal and L. Bosio, J. Chem. Phys., 98, 4246 (1993).

[9] R. Guégan, D. Morineau, R. Lefort, W. Béziel, M. Guendouz, L. Noirez, A. Henschel and P. Huber, Eur. Phys. J. E, 26, 1292 (2008).

[10] Y. Xia, G. Dosseh, D. Morineau and C. Alba-Simionesco, J. Phys. Chem. B 110, 19735 (2006).

[11] F. Bruni, M. A. Ricci and A. K. Soper, J. Chem. Phys. 109, 1478-1485 (1998); A. K. Soper, F. Bruni and M. A. Ricci, ibid. 109, 1486-1494 (1998).

[12] D. Morineau and C. Alba-Simionesco, J. Chem. Phys., 118, 9389 (2003).

[13] D. Morineau, R. Guégan, Y. Xia and C. Alba-Simionesco, J. Chem. Phys. 121, 1466-1473 (2004).

[14] M. Bée, Quasielastic Neutron Scattering, Adam Hilger, Bristol, (1988).

[15] D. T. Bowron, J. L Finney, and A. K. Soper, Mol. Phys. 93, 531 (1998). 
[16] D. Morineau, C. Alba-Simionesco, M. C. Bellissent-Funel, and M.-F. Lauthié, Europhys. Lett. 43, 195, (1998).

[17] D. Morineau and C. Alba-Simionesco, J. Chem. Phys, 109, 8494 (1998).

[18] D. Morineau and C. Alba-Simionesco, J. Phys. Chem. Lett., 1, 1155-1159 (2010).

[19] J. Wuttke, W. Petry, G. Coddens and F. Fujara, Phys. Rev. E 52, 4026 (1995).

[20] O. Sobolev, A. Novikov, and J. Pieper, Chem. Phys., 334, 36 (2007).

[21] H. Sillescu, J. of Non-Cryst. Solids 243, 81 (1999).

[22] F. He, L.-M. Wang, and R. Richert, Phys. Rev. B 71, 144205 (2005).

[23] P. Scheidler, W. Kob and K. Binder, Europhys. Lett. 59, 701 (2002).

[24] C. J. Ellison and J. M. Torkelson, Nature Mater. 2, 695 (2003).

[25] R. Busselez, R. Lefort, Q. Ji, F. Affouard, and D. Morineau, Phys. Chem. Chem. Phys., 11, 1112711133, (2009).

[26] Q. Ji, R. Lefort, and D. Morineau, Chem. Phys. Lett., 478, 161-164 (2009).

[27] T. Bellini, L. Radzihovsky, J. Toner, and N.A. Clark, Science, 294, 1074-1079 (2001).

[28] A. V. Kityk, M. Wolff, K. Knorr, D. Morineau, R. Lefort, and P. Huber, Phys. Rev. Lett. 101, 187801 (2008).

[29] R. Guégan, D. Morineau, C. Loverdo, W. Beziel and M. Guendouz, Phys. Rev. E 73, 011707 (2006).

[30] G. Chahine, A. V. Kityk, N. Desmarest, F. Jean, K. Knorr, P. Huber, R. Lefort, J. M. Zanotti, and D. Morineau, Phys. Rev. E, 82, 011706 (2010).

[31] Z. Kutnjak, S. Kralj, G. Lahajnar, and S. Zumer, Phys. Rev. E 68, 021705 (2003).

[32] Q. Ji, R. Lefort, A. Ghoufi and D. Morineau, Chem. Phys. Lett., 482, 234-238 (2009).

[33] R. Lefort, D. Morineau, R. Guégan, M. Guendouz, J.M. Zanotti, and B. Frick, Phys. Rev. E 78, 040701 (2008).

[34] R. Guégan, D. Morineau, R. Lefort, A. Moreac, W. Béziel, M. Guendouz, J.M. Zanotti, and B. Frick, J. Chem. Phys. 126, 064902 (2007).

[35] G.P. Sinha, and F.M Aliev, Phys. Rev. E 58, 2001-2010 (1998).

[36] R. Lefort, D. Morineau, R. Guégan, C. Ecolivet, M. Guendouz, J.-M. Zanotti, and B. Frick, Phys. Chem. Chem. Phys. 10, 2993 (2008). 